\title{
UPSCALING TRANSMISSIVITY IN THE NEAR-WELL REGION FOR NUMERICAL SIMULATION: A COMPARISON ON UNCERTAINTY PROPAGATION
}

\author{
Jianlin $\mathrm{Fu}^{*{ }^{*+}}$, Carl L. Axness*† and J. Jaime Gomez-Hernandez* \\ * Department of Hydraulic and Environmental Engineering, Technical University of Valencia, \\ 46022, Valencia, Spain \\ +E-Mail:fu_jianlin_ac@yahoo.com (Corresponding Author) \\ \# Department of Energy Resources Engineering, Stanford University, 367 Panama Street, \\ Stanford, CA 94305, USA \\ † Sandia National Laboratories, Albuquerque, USA
}

\begin{abstract}
Upscaling transmissivity near the wellbore is expected to be useful for well performance prediction. This article ascertains the need of upscaling by comparing several numerical schemes and presents an approach to upscale transmissivity in the near-well region. This approach extends the Laplacian method with skin, which was successfully applied to the parallelepiped flow case, to radial flow case in the vicinity of wellbore through a nonuniform gridding technique. Several synthetic fields with different stochastic models are chosen to check the efficiency of this method. Both flow and transport simulations are carried out in finite heterogeneous confined aquifers to evaluate the results. It is demonstrated that the proposed method improves the ability of predicting well discharge or recharge and solute transport on the coarse scale in comparison with other schemes by examining the uncertainty propagation due to upscaling.
\end{abstract}

Keywords: geostatistics, well capture zone, radial flow, stochastic model, reservoir simulation, gridding

\section{INTRODUCTION}

Stochastic modeling of reservoir parameters with the aid of geostatistical techniques can effectively provide high-resolution models of reservoir at the measurement scale (Fu and Gomez-Hernandez, 2008 and 2009a). Limitation in computer resources forces these models to be upscaled to a modeling (coarse) scale such that a numerical simulator can afford in practical engineering applications of computational fluid mechanics, e.g., petroleum engineering, hydrogeology, environmental engineering, $\mathrm{CO}_{2}$ sequestration, etc. (see Li et al., 2007; Fu, 2008; Jenny et al., 2003). A large number of upscaling approaches have been developed to coarsen detailed aquifer or reservoir models into those at an appropriate scale for numerical simulations (Wen and GomezHernandez, 1996; Renard and de Marsily, 1997). Many of them are proven quite efficient for upscaling under the uniform flow condition, where the local piezometric head or pressure values vary normally slowly, or say "linearly" (Durlofsky et al., 2000).

For the immediate vicinity of a well, however, these existing upscaling approaches may not completely apply due to the fact that the flow pattern is no longer uniform but convergent around a pumping well or divergent near an injection well. The pressure gradient typically increases close to the well and becomes highly sensitive to the spatial variation of hydraulic conductivity (Desbarats, 1992; Fiori et al., 1998) and especially to the difference between the global mean conductivity and the value at the wellbore (Axness and Carrera, 1999). Moreover, the distribution of concentration and the breakthrough curve of conservative tracers are typically different from those of the uniform flow cases. An effective upscaling scheme should be able to capture this character of pressure gradient distribution around the well and honor the statistical structure of conductivity in order to provide accurate coarse models. Basically, there are two problems needed to be addressed for upscaling: (1) Can the coarse grid account for the flow geometry in the near-well region? and (2) Can block transmissivity adequately honor heterogeneities of the aquifer or reservoir?

As for the first problem, several authors have already presented some approaches to address it. Ding (1995) proposed an upscaling procedure which consists of upscaling transmissivity and numerical productivity index. An obvious 
improvement over traditional methods is observed. Durlofsky et al. (2000) further extended Ding's technique to the 3D case. Muggeridge (2002) assessed Ding's method in a variety of case studies with partially penetrating wells and nonvertical wells of both two- and three-dimensional problems. Wolfsteiner and Durlofsky (2002) developed an upscaling approach for a near-well radial grid on the basis of the so-called multiblock-grid simulation technology. Such grid is globally unstructured but maintains locally structured. However, a drawback of them is that they use a regular coarse grid, either rectangular or almost rectangular, although a non-uniform grid is applied among them, e.g., Durlofsky et al. (1997), which is considered to be efficient for dealing with the case of connected region with high conductivity values. The influence of regular gridding lies in that once the simulation grid becomes very coarse or the upscaling ratio is quite high, a significant or even intolerable loss of information will arise. That is because the regular coarse grid has a rather limited flexibility to capture the feature of pressure gradient variation near the wellbore. An improvement in grid design and/or refinement is expected to enhance the accuracy of upscaling under the condition of preserving the coarsening ratio, or upscaled factor, so as to produce a coarsened model at a reasonable scale as the input to the flow simulator. The second problem is concerned about the computation of the equivalent transmissivity for the coarse-scale model based on the fine-scale model. It is known that using the upscaled transmissivity for the coarse-scale flow simulation is much more accurate than the upscaled conductivity (e.g., Jenny et al., 2003). It is also well known that the block transmissivity is not only an intrinsic property of the porous media, but also depends on the flow geometry and boundary conditions (e.g., Gomez-Hernandez and Journel, 1994). Typical numerical approaches to computing the equivalent or block transmissivity call for solving flow problems over the local finescale block, which includes all the cells that are embedded in the corresponding coarse grid. One of the most striking considerations is the configuration of boundary conditions for the coarse grid (e.g., Jenny et al., 2003; Fu et al., 2010). This is of paramount importance because different boundary specifications will produce quite distinct equivalent block values. White and Horne (1987) computed the block conductivity from the solutions of flow for several alternative boundary conditions. Gomez-Hernandez and Journel (1994) applied a skin surrounding the coarse grid as the boundary configuration, which accounts for the influences from the neighbor cells but without resorting to solve flow problems over the entire field. Durlofsky (1991) developed an approach to yield a set of symmetric, positivedefinite block conductivity tensors by applying periodic boundary conditions. All these methods assume that the block transmissivity is a local or extendedly local property of porous media, i.e., the effects of neighbors can be ignored. Global approaches, on the other hand, consider such influences by solving flow problems on the global fine scale, e.g., Holden and Nielsen (2000). However, this method may be extremely computationally expensive. More recently, to overcome this shortcoming, Chen et al. (2003) developed a technique that couples local and global approaches with the aid of iterative solutions to flow and/or transport problems on the global coarse scale and the local fine scale.

In addition, an extra technical detail is how to choose a neighbor size for solving local flow problems with specified boundary conditions. That is, should the neighbor cells be included when solving local fine-scale flow problems? Mascarenhas and Durlofsky (2000) proposed a near-well upscaling method extending the local fine regular grid to include neighbor regions when the transmissivity tensors are computed for a coarse regular grid, while a traditional upscaling procedure does not do so. Their numerical results from single- and multi-phase flow experiments display a significant improvement compared to the conventional methods by analyzing inflow profile and water cut parameters based on the fine-scale simulation against those based on the coarse-scale simulation. Actually, a similar enhancement was observed when upscaling transmissivity for the uniform flow, where the Laplacian method with skin (Gomez-Hernandez and Journel, 1994) or border region (Wen et al., 2003; Chen et al., 2003) is named. In the present study, we include this idea into our upscaling approach with a slight modification to the coarse radial grid.

This paper proceeds as follows: the next section gives details of the proposed upscaling method. Then, we outline the assessment criteria for subsequent comparisons and the numerical methods for flow and transport simulations. In the fourth section, the numerical results are compared with several existing upscaling techniques. It follows by some discussion on the upscaling techniques. Finally, the paper ends up with a summary of the proposed method for upscaling transmissivity in the near-well region. 


\section{UPSCALING}

An upscaling procedure in numerical simulation typically consists of three steps: first, using geostatistical techniques, a series of fine, detailed model parameters (i.e., hydraulic conductivity) are generated, each representative of the geology and hydrology of the area. Then a coarse grid is designed to capture main characteristics of flow and transport accordingly at the coarse scale. Finally, an equivalent value, either scalar or vectorial, calculated from the fine model of scalar parameters is assigned to the coarse model.

\subsection{Generation of conductivity fields at the fine scale}

The currently existing geostatistical techniques allow for generating property fields of reservoir at a point scale or for a uniform grid. The former can generate parameter fields of reservoir at any location for an arbitrary grid. This is a simple but fast scheme since only a small quantity of data are necessary for the near-well region. Moreover, it circumvents the scaling problem. The deficiency, however, lies in that it can not capture the detailed spatial variation of reservoir parameters. The latter, on the contrary, generates data at the specified position in a regular grid frame. This requires a very fine grid for the entire field such that the spatial fluctuations in the near-well region are adequately represented. However, it inevitably brings up a scaling problem in order to produce a proper coarse model as input to the expensive flow simulator. For the purpose of checking the necessity of upscaling in the near-well region, we compare these two types of techniques subject to an identical flow and transport scenario.

The sequential simulation algorithm is a powerful stochastic simulation technique and has been applied in many studies (Gomez-Hernandez and Journel, 1993). It can be used to generate conditional or unconditional realizations from either multi-Gaussian or non-Gaussian random functions. Following the two-point geostatistics, the multi-Gaussian hydraulic conductivity field, $\ln K(\mathbf{x})$, is modeled through a normally distributed random space function, $Y(\mathbf{x})=\ln K(\mathbf{x})$, with an exponential semivariogram specified by,

$\gamma_{Y}(r)=\sigma_{Y}^{2}\left[1-\exp \left(-r / \lambda_{Y}\right)\right]$

where $r$ is the two-point separation distance, $\sigma_{Y}{ }^{2}$ is the variance, and $\lambda_{Y}$ is the correlation length. For the non-Gaussian model, the indicator semivariogram for a continuous variable is defined as, ind $_{i}=1\left(\right.$ if $Y_{i} \leq$ cut $\left._{k}\right)$;

ind $_{i}=0$ (if $Y_{i}>c u t_{k}$ ),

where the subscript $i$ refers to a particular location, and the cutoff $\mathrm{cut}_{k}$ is a threshold that is specified for the $k$ th class of the continuous variable $Y$ to create the indicator transform.

Once the semivarigram matrix is specified, the corresponding covariance is easily obtained through $C(r)=C(0)-\Gamma(r)$ if the lag effect is ignored and the stochastic samples may be efficiently generated by the sequential simulation approach. The public domain codes GCOSIM3D (GomezHernandez and Journel, 1993) and ISIM3D (Gomez-Hernandez and Srivastava, 1990) are used to generate hydraulic conductivity fields at the fine scale. Fig. 1 gives three typical realizations of log-conductivity field with $801 \times 801$ square cells: Fig. 1(a) is a multiGaussian field with statistically isotropic structure, Fig. 1(b) is a multi-Gaussian field with statistically anisotropic structure, and Fig. 1(c) is a non-Gaussian field with statistically anisotropic structure. Each cell has a dimension of $0.25 \times 0.25$. The wellbore is assumed to locate at the center of the computational domain, i.e., with the planar coordinate in index $(401,401)$. We assume the property value at the wellbore center is known, so the generation of log-conductivity field belongs to conditional simulation.

Due to the limitation of GCOSIM3D, however, the existing code has no ability of generating data with arbitrarily irregular grids although the sequential simulation algorithm may allow one to do so in theory. But we can address this problem by resampling from the fine-scale field. That is, we first generate a fine-scale field by assuming a statistical structure, and then resample the property values from this field and assign them to proper locations in the irregular grid. The resampled field shares the same statistical structures as that of the fine scale, even though it ignores some details of the spatial variations. One of the advantages of this way is that its results can be used to compare with those of responding field directly. We denote it as a non-upscaled field.

\subsection{Design of the coarse grid}

The Thiem solution to a 2D steady-state head field in a homogeneous medium with prescribed heads at the well radius and at an exterior circular boundary can be written as,

$h(r)=h_{w}+\left(h_{e}-h_{w}\right) \ln (r) / \ln \left(r_{e} / r_{w}\right)$, 
InT field realization no.1

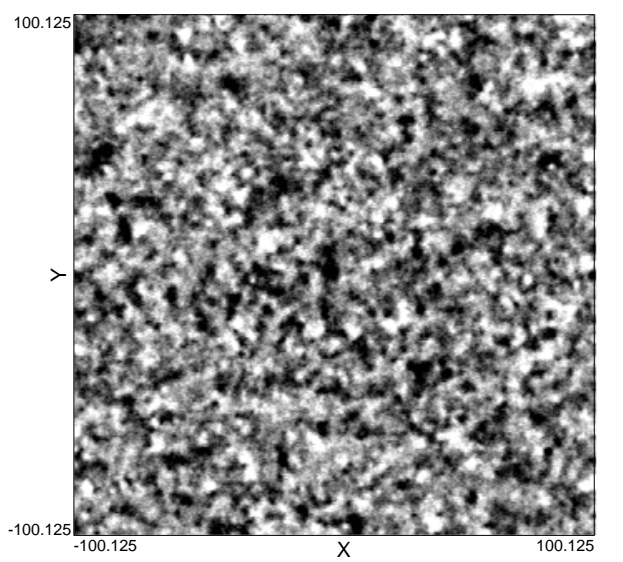

(a)

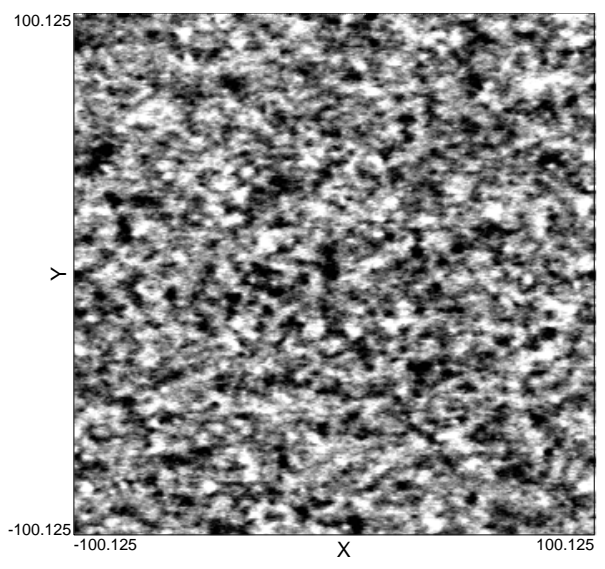

(b)

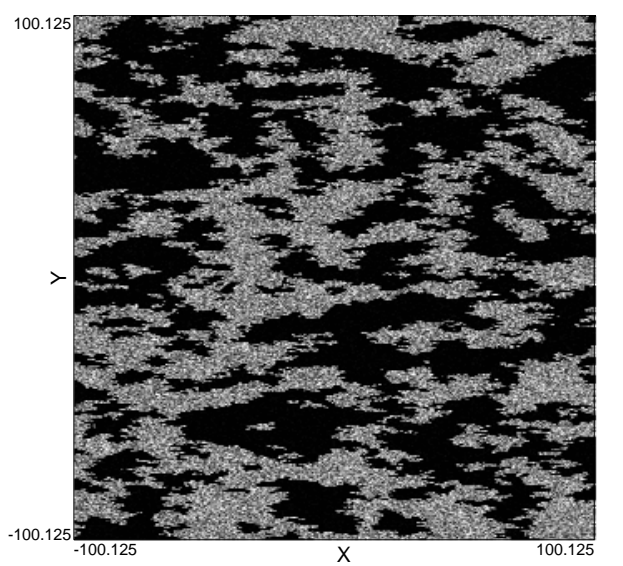

(c)

Fig. 1 Several typical realizations of transmissivity field: (a) a multi-Gaussian field with isotropic structure, (b) a multi-Gaussian field with anisotropic structure, and (c) a non-Gaussian field with anisotropic structure.

where $r$ represents the normalized radius $r=r^{\prime} / r_{w}$; $r_{w}$ is the wellbore radius; $r^{\prime}$ is the radius away from the well axis; $h_{e}$ and $h_{w}$ are the heads at the outer and inner radii, respectively. Although this solution is only applicable to homogeneous media, it may be shown that gridding with respect to $\ln (r)$ other than $r$ minimizes the error in the resulting hydraulic head (Axness et al., 2004). The coarse grid design in terms of log-scale, therefore, will have more advantages than those of the natural scale. The former is expected to be able to capture the main features of gradient variations better than the latter even for highly heterogeneous media.

In this study, we first normalize the well radius to one unit, i.e., $r_{w}=1$, while the exterior radius is equal to 100 units, i.e., $r_{e}=100$. The whole circular field is divided into ten annuli excluding the well block. The ratio of radius increment of every annulus compared to that of the previous inner annulus is 1.584893 , i.e., $\Delta r_{i+1} / \Delta r_{i}=1.584893$. Those rings are further divided into twelve segments along the ray direction. This ensures the grid is very fine close to the wellbore but quite coarse far away from it (see Fig. 2(a)). The original $801 \times 801$ fine square grid is upscaled into a $12 \times 10$ coarse radial, circular grid. In order to validate the efficiency of this grid design, we have compared its results with those of equal radius increment (Fig. 2(b)).

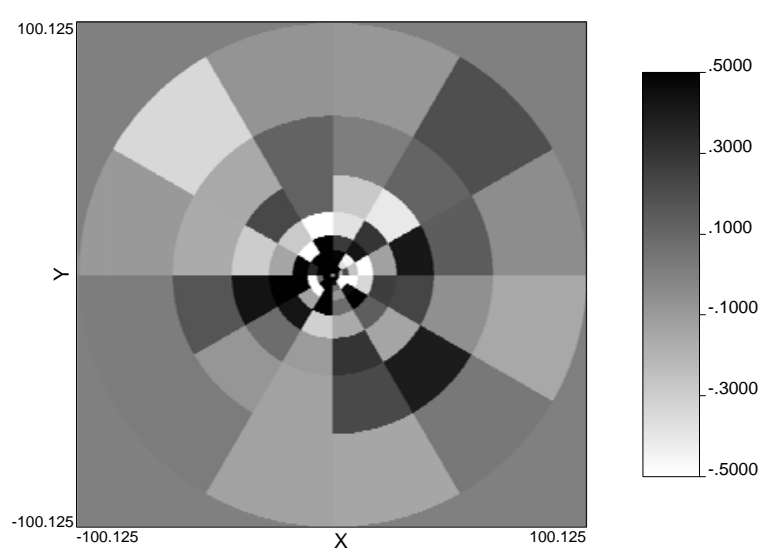

(a) InT field in non-uniform coarse grid.

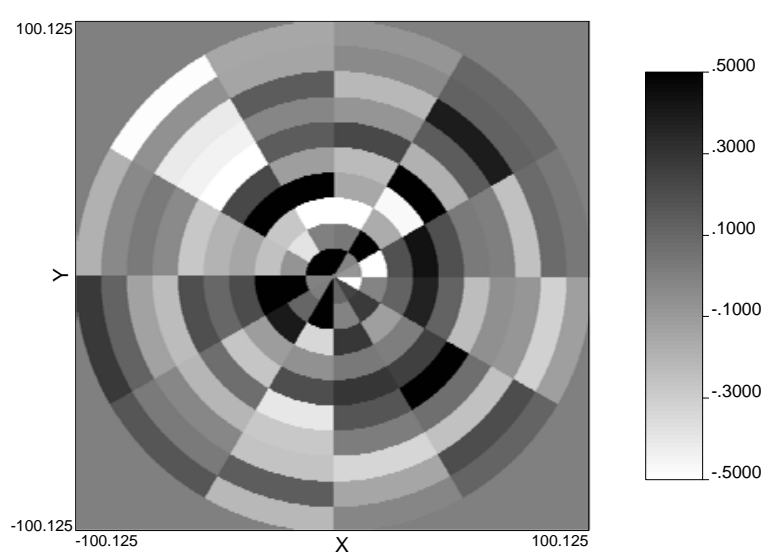

(b) InT field in uniform coarse grid.

Fig. 2 Geometry of the coarse grid: (a) non-equal radius increment and (b) equal radius increment. 


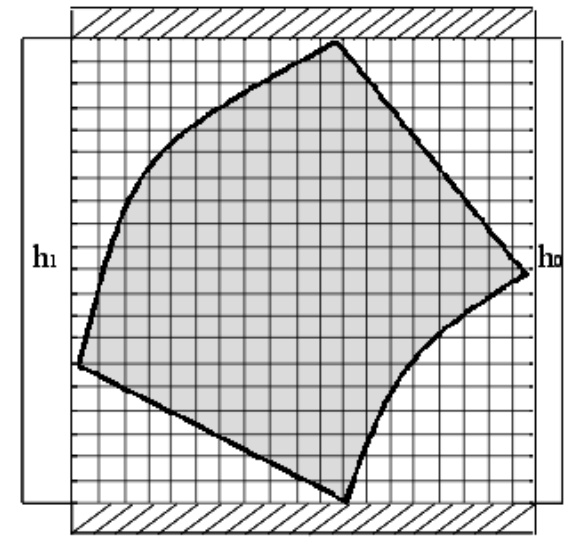

(a) Computation of Tv,xx

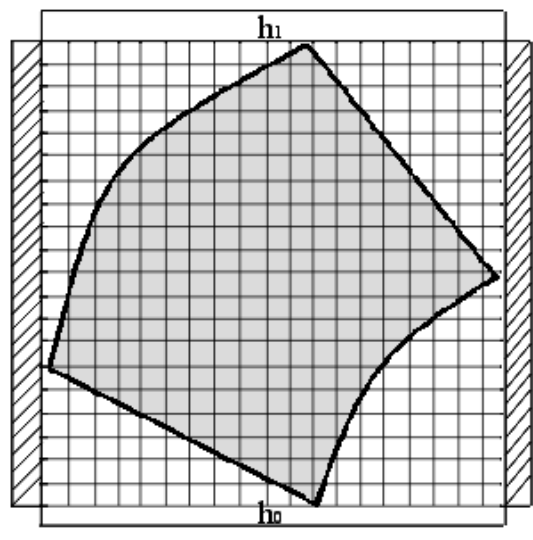

(b) Computation of Tv,yy

Fig. 3 Configuration of boundary conditions for the coarse grid: (a) $T_{V, x x}$, (b) $T_{V, y y \text {. }}$

\subsection{Computation of equivalent transmissivity}

We extend the concept of skin (GomezHernandez and Journel, 1994) to the computation of equivalent transmissivity for the coarse grid blocks. Two sets of boundary conditions are considered for each coarse block: one is in the $x$ direction and the other in the $y$ direction (Fig. 3). By doing so, we can obtain the $T_{x x}$ and $T_{y y}$ correspondingly through solving (extendedly) local flow problems at the fine scale. In addition, one of the crucial problems is the configuration of boundary conditions for each coarse rectangular block. We approximate the head values for each side by assigning a Thiem solution to the middle point of each edge such that the computationally demanding global problem for boundary condition configuration is avoided but a relatively accurate result is still achieved. The method developed here closely follows the seminal idea of the work that was presented in a conference paper (Fu et al., 2006).

The procedure for calculating the equivalent transmissivity is as follows (Fig. 3):

1. Define the rectangular block $R$ that includes the non-rectangular target block $B$;

2. Solve the flow problem with a specified boundary condition system which consists of non-flow boundary and prescribed head. The prescribed heads are approximated by the analytical solution for the homogeneous media as described as above;

3. Evaluate the average flow rate $Q_{V}$ and the average head gradient $\Delta h_{V}$ over the nonrectangular target block $B$ both in $x$ and $y$ directions;

4. Compute the equivalent transmissivity by,

$$
\begin{aligned}
& \mathrm{T}_{\mathrm{V}, \mathrm{xx}}=-\mathrm{Q}_{\mathrm{V}, \mathrm{xx}} / \Delta \mathrm{h}_{\mathrm{V}, \mathrm{xx}}, \\
& \mathrm{T}_{\mathrm{V}, \mathrm{yy}}=-\mathrm{Q}_{\mathrm{V}, \mathrm{yy}} / \Delta \mathrm{h}_{\mathrm{V}, \mathrm{yy}} .
\end{aligned}
$$

\section{NUMERICAL SIMULATION}

We choose two types of parameters for the assessment of upscaled results: one is water injections (or, similarly, yields for a pumping well) into the wellbore, and the other is travel time of conservative tracers. The former can be achieved by calculating the flow rate in the wellbore after solving flow equations. The latter can be accomplished by solving transport equations with the aid of the random walk particle tracking method.

\subsection{Flow and transport simulation}

The flow problem is solved by tailoring the blockcentered finite-difference simulator to the radial flow case (Fu, 2008). To avoid the discretization error, all the simulations are performed at the fine scale. The transmissivity at the interface between cells are computed using harmonic averages of the adjacent blocks. We model the radial flow to a well by specifying fixed heads at the wellbore and at the exterior circular boundary, i.e., $h_{w}=10$ and $h_{e}=0$, respectively. It models the case of an injection well or source configuration since the pressure at wellbore is higher than that of the surroundings.

We prefer an injection well to a pumping well on the basis of the consideration that the latter will have a rather lower particle capture rate when we employ the particle tracking scheme to solve transport problems, which will undermine the reliability of solutions. Our experience shows that characteristics of flow and transport around an injection well have no obvious difference from those surrounding a pumping well. Second, we 
intentionally impose a prescribed head boundary at the exterior radius since we reason that its influence is not significant when the outer radius is placed a few correlation lengths, e.g., ten as in this study, away from the well (Axness and Carrera, 1999). Finally, we choose a constant head at the wellbore as an evaluation criterion not only because it is widely used in the engineering practice but also because it is the easiest way to modeling the steady flow.

The transport equation solver adopts the random walk particle tracking scheme developed by $\mathrm{Fu}$ (2008). Two thousand particles released from the wellbore are followed until they arrive at the control circle, which is set 100 units away from the wellbore. Because the pressure near the wellbore is higher than that far away, it ensures that all particles move from the wellbore to the exterior boundary. It evidently overcomes the difficulty of rather lower particle capture rate, which is common in employing the particle tracking scheme to solve the transport equations. By doing so, a stable solution can be obtained.

\subsection{Computation of well discharge/recharge}

In this study, the well injection is calculated by numerically integrating Darcian velocity along the surface surrounding the wellbore,

$Q_{w}=\int q(\theta) \mathrm{d} \theta$,

where $q(\theta)$ is the component of Darcian velocity around the wellbore. To ease the complexity of problems, we assume that property values at the wellbore are the same as those at the well block.

\subsection{Computation of travel time}

Well discharge or recharge reasonably reflects an average effect of heterogeneities in porous media. But it can not sufficiently display the variation of heterogeneity. Travel time of tracers computed by particle tracking scheme has a more powerful ability to do so. This is because the evolution of a solute plume is more sensitive to perturbations of the hydraulic conductivity and head field. There exist two ways to evaluating the upscaling approaches in terms of travel time: (1) compare travel time of single realization to check their accuracy; and (2) compare several realizations to check their robustness. We adopt both of these two methods. Due to the difficulty of comparing the whole breakthrough curve for all realizations, we only sample several typical points from the breakthrough curve (BTC), e.g., $t_{5 \%}, t_{25 \%}, t_{50 \%}$ and $t_{95 \%}$, which account for the early, middle and late arrival time.
It is worth emphasizing that the selection of the most appropriate part of BTC is of importance in engineering environmental operations, e.g., to monitor the extent and degree of groundwater contamination from a known source ( $\mathrm{Fu}$ and Gomez-Hernandez, 2009b). $t_{5 \%}$ better reproduces the earliest part of the reference BTC and represents the fastest particles arriving at the control plane as needed for the design of radioactive underground repository. The earliest arrivals in the BTC follow the fastest pathways between the release source and the control plane, which are dominated by preferential flow and reactive transport paths. Failing to account for such cases will yield a too conservative conclusion in risk analysis in that the real arrival time may be much faster than that estimated (Gomez-Hernandez and Wen, 1998). $t_{25 \%}$ and $t_{75 \%}$ reproduce the middle part of the reference BTC and reflect the portion of particles arriving to the control plane with high frequency. Public officials assessing health risks associated with contaminant exposure in a drinking water supply system may be most concerned with this parameter. $t_{95 \%}$ reproduces the tail part of the reference BTC and denotes the slowest particles arriving to the control plane as needed for mass removal calculations in remediation problems. The late travel times reflect a more integral behavior, or even flow and reactive transport barriers. An aquifer remediation design without considering such feature may fail because the resident contaminants will be removed more slowly than expected (Wagner and Gorelick, 1989).

\section{RESULTS}

Four types of coarsening techniques are assessed in this section: (a) the proposed scheme as previously stated (named proposal), (b) traditional geometric average from the fine scale in the framework of non-uniform grid (called GM), (c) non-upscaled method (abbreviated to NP), and (d) the geometric average from the fine scale in the framework of uniform grid (shortened to UG). The second one, GM, assigns the coarse conductivity to be the geometric mean of support cells contained in the coarse element. This is the simplest and traditional method for upscaling uniform flow. We present the results here with two aims: one is to check its efficiency under the radial flow conditions, and the other is to compare its results with the proposed approach. The third one, NP, simply assigns the conductivity in the coarse element to be the point value at the coarse element centroid or to be the conductivity of the 
support scale element closest to the centroid when the field is generated at a fine support scale. The last technique, UG, is done the same way as the second but only distinctly with uniform grid intervals.

Flow and transport results from the four types of techniques are presented in three scenarios: the first is multi-Gaussian conductivity fields with isotropic structures; the second is multi-Gaussian conductivity fields with anisotropic structures; and the last one is non-Gaussian conductivity fields with anisotropic structures.

\subsection{Multi-Gaussian fields with isotropic structures}

One hundred $\ln K(\mathbf{x})$ fields are generated by GCOSIM3D, each of them with a correlation length equal to ten in both $x$ and $y$ directions, i.e., $\lambda_{x}=\lambda_{y}=10$, and the expected variances more than one, i.e., $\sigma_{\ln K(\mathbf{x})}{ }^{2}=2$. The $\ln K(\mathbf{x})$ fields are scalar, that is, the conductivity value in the $x$ direction are the same as those in the $y$ direction, i.e., $\ln K_{x x}=\ln K_{y y}$. The mean of $\ln K(\mathbf{x})$ is set to zero, i.e., $\mathrm{E}[\ln K(\mathbf{x})]=0$. The flow rates of the wellbore are computed on the fine scale named reference

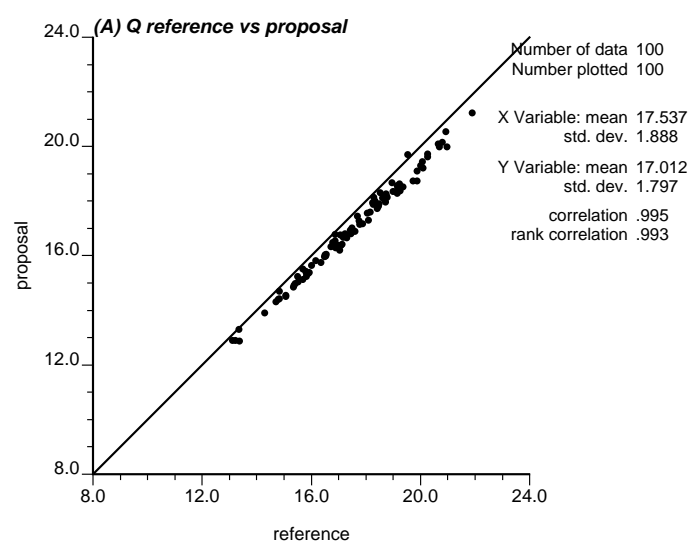

(a) Q reference vs proposal

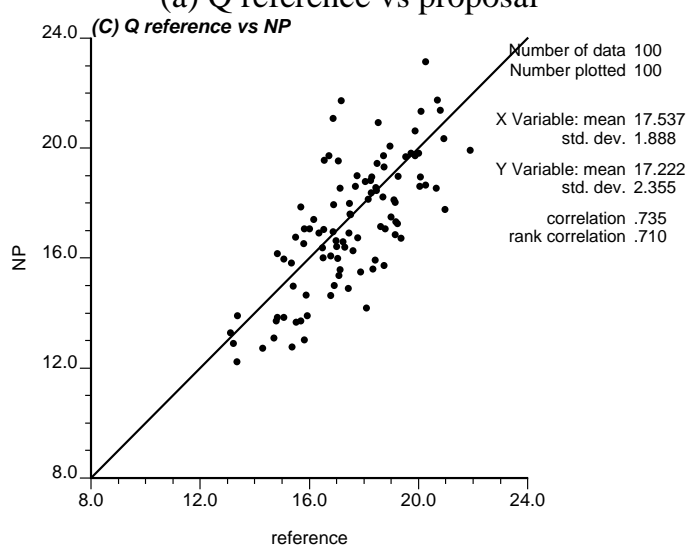

(c) Q reference vs NP values. Then, the fine-scale fields with $801 \times 801$ grids are upscaled to those with $12 \times 10$ coarse grids in the same way as described above. The fluxes over the wellbore are computed at the coarse scale for four different coarsening approaches, namely proposal, GM, NP and UG.

Fig. 4 shows the relationship of wellbore fluxes between the reference fluxes and those using the four different approaches. Fig. 4(a) displays one hundred wellbore fluxes by the proposed upscaling approach compared to those of reference fine scales. The average flux of one hundred realizations is 17.537 for reference fields and 17.012 for the proposal method with relative error of only 3\%. It shows that the upscaled values are a rather reasonable approximation. Note, moreover, that the $x$ and $y$ mean values are close to each other, meaning that this method is unbiased, that is, the upscaled Q tends to be close to the reference $\mathrm{Q}$ in the mean. Fig. 4(b) shows the performance of the traditional geometric mean method. The correlation and rank correlation coefficient have a slight decrease but still more than $99 \%$. It seems that the geometric mean method is quite efficient and robust in upscaling radial flow in the near-well region for a scalar

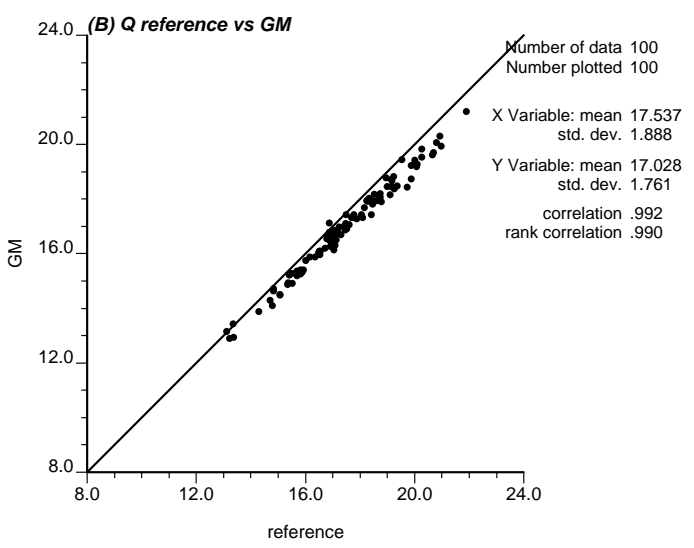

(b) Q reference va GM

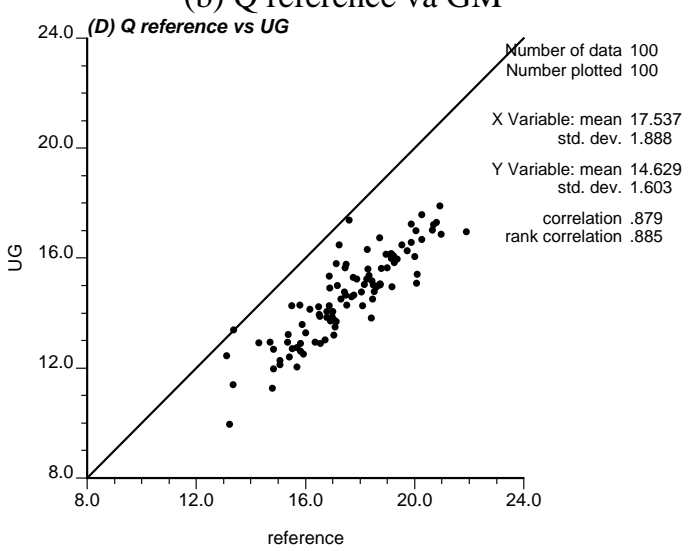

(a) Q reference vs UG

Fig. 4 Wellbore fluxes cross relationship between the fine scale and the coarse scale of multi-Gaussian transmissivity fields with isotropic structures. 


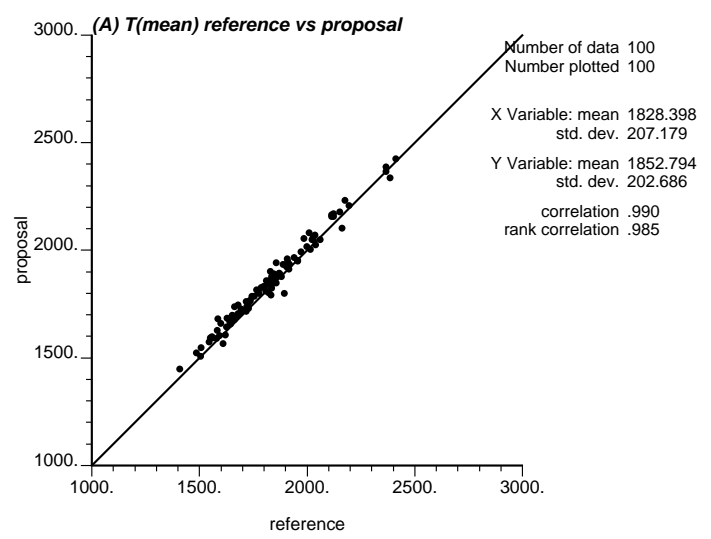

(a) T(mean) reference vs proposal

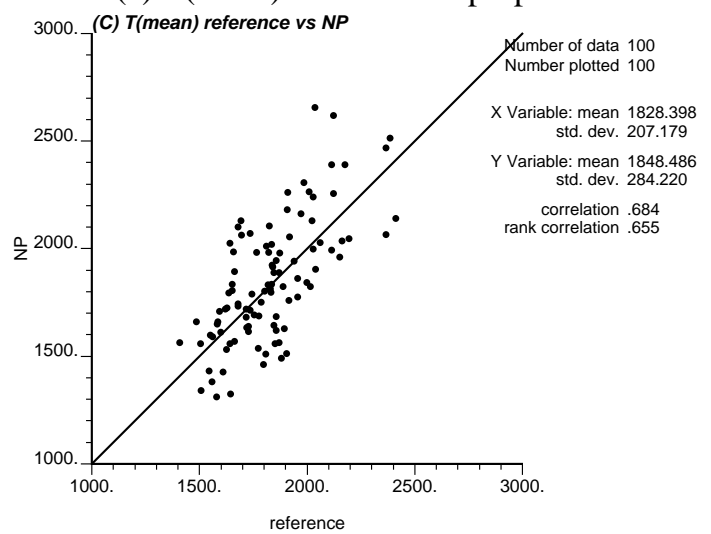

(c) T(mean) reference vs NP

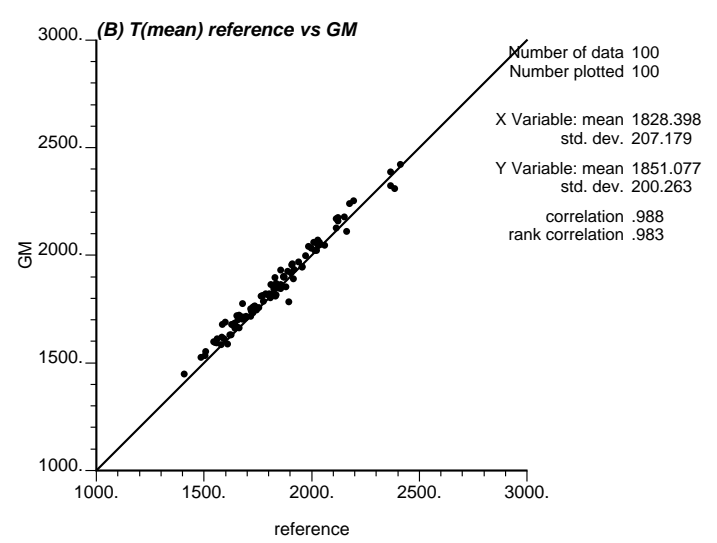

(b) T(mean) reference vs GM

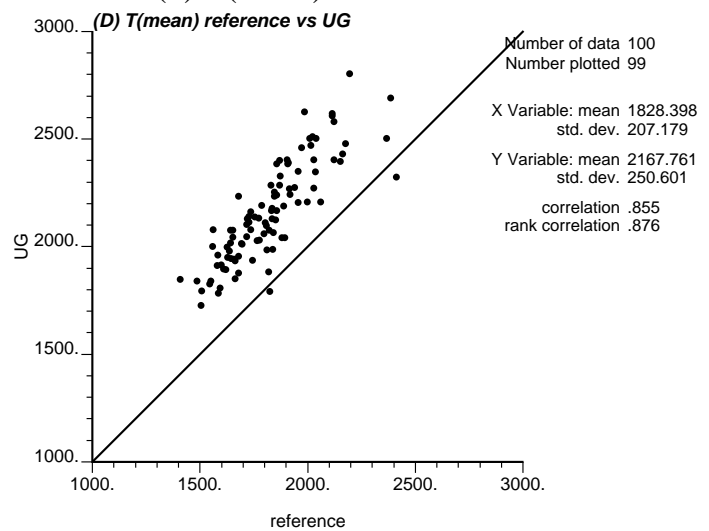

(d) T(mean) reference vs UG

Fig. 5 Average breakthrough time cross relationship between the fine scale and the coarse scale of multi-Gaussian transmissivity fields with isotropic structures.

log-conductivity field with isotropic structures. Fig. 4(c) plots the result of the NP method. The correlation coefficient is only $73 \%$. Obviously the reproduction ability is worse than that of the GM method. Fig. 4(d) is the result of the UG method, i.e., that with a uniform interval grid. The wellbore fluxes calculated from the coarse grid are severely deviated from those of the fine grid. Although the correlation coefficient is up to $88 \%$, the mean of wellbore fluxes has a rather high error of up to $20 \%$. Such apparent error is intolerable for the computation of well yields.

The similar average effect of upscaled heterogeneities can be observed from the mean breakthrough time after solving solute transport problems. Fig. 5 plots the average breakthrough times of different coarse fields versus those of fine reference fields. Fig. 5(a) shows the average breakthrough times of one hundred realizations calculated by the proposed upscaling approach. Their correlation coefficient with those of reference fields is up to $99 \%$ and the relative average error is only $1.3 \%$. The GM method also provides rather acceptable results as shown in Fig. 5(b). The relative average error is $1.2 \%$ and the correlation coefficient is a little bit worse but still more than 98\%. The NP method produces unpleasant results as shown in Fig. 5(c). Although the average error is quite low only with $1 \%$ owing to the randomness of sampling, the solutions are too unstable with the correlation coefficient of only $68 \%$. The UG method has the results with a better correlation coefficient of almost of $85 \%$, but the average error is up to $16 \%$. In summary, the first two upscaling schemes produce quite satisfactory results.

Unlike the well discharge or recharge and the average breakthrough time, which are only overall effects of field fluctuations, a whole breakthrough curve can provide a better review of upscaling results because it can adequately sample the spatial variation of heterogeneous fields. Fig. 6 gives a comparison of five breakthrough curves from a typical realization. The results from the NP and UG methods obviously deviate from that of the reference fine scale. The proposed approach has a slightly better capability of reproduction than the GM method. For this single realization, the proposed method produces better results at the early and middle arrival time.

In order to further inspect the stability of the proposed approach in reproducing the 
breakthrough curve of reference fields, we sample four typical points, i.e., $t_{5 \%}, t_{25 \%}, t_{75 \%}$, and $t_{95 \%}$, from the breakthrough curve and compare them with those of the GM method. Fig. 7 illustrates the comparison of the breakthrough curve matching with reference fields between these two methods. The left column plots the matching of the proposed method from all one hundred realizations, and those of the GM method are listed in the right column. As for the average breakthrough error, the proposed approach gains some advantage over the GM method: almost all four sample points of the former have closer values to the reference ones than those of the latter. Moreover the stability of the latter has no diminishment in general; their correlation coefficients maintain at about $90 \%$.

\subsection{Multi-Gaussian fields with anisotropic structures}

One hundred $\ln K(\mathbf{x})$ fields are generated by GCOSIM3D, each of them with a correlation length equal to ten in the $x$ direction and five in the $y$ direction, i.e., $\lambda_{x}=10$ and $\lambda_{y}=5$. Their expected variances are kept more than one, i.e., $\sigma_{\ln K(\mathbf{x})}{ }^{2}=2$. The $\ln K(\mathbf{x})$ fields are constant vectorial, that is, the $\ln K(\mathbf{x})$ values in the $y$ direction are one half of those in the $x$ direction, i.e., $\ln K_{x x}=\ln K_{y y} / 2$. The mean of $\ln K(\mathbf{x})$ is set to zero, i.e., $\mathrm{E}[\ln K(\mathbf{x})]=0$. The fluxes over the wellbore are computed for the fine-scale field and four different coarse fields in the same manner as in the first scenario.

Fig. 8 shows the cross relationship of the wellbore fluxes between the reference and four different upscaling approaches. Fig. 8(a) exhibits one hundred wellbore fluxes via the proposed upscaling approach with comparison to those of the reference fine scales. The average flux of one hundred realizations is $\mathbf{1 2 . 4 6 9}$ for reference fields and 12.077 for the proposed method with a relative error of only 3\%. It shows that the upscaled values are quite close to the actual case. Moreover, the correlation and rank correlation coefficient between two types of fluxes are more than $99 \%$, which demonstrates the robustness of this approach. Fig. 8(b) indicates the performance of the traditional geometric mean method. The correlation and rank correlation coefficient have a little decrease but still close to 99\%. Fig. 8(c) plots the result of the NP method. The correlation coefficient is only $71 \%$. Fig. 8(d) is the results of the UG method, which obviously underestimates the actual values. The mean of wellbore fluxes has a rather high error up to $19 \%$, although the correlation coefficient reaches $88 \%$.

Fig. 9 plots the average breakthrough times of different upscaled fields versus those of reference fields with anisotropic structures. Fig. 9(a) shows the average breakthrough times of one hundred realizations from the proposed upscaling approach. The correlation coefficient with those of the reference fields is up to $99 \%$ and the relative average error is only $1.3 \%$. The GM method also provides acceptable results as shown in Fig. 9(b). The relative average error is $1.2 \%$ and the correlation coefficient is a little bit worse but still more than $98 \%$. The NP method produces unpleasant results as in Fig. 9(c). Although the average error is only $1 \%$ owing to the randomness of sampling, the stability is too low: the correlation coefficient is only 68\%. The UG method has results with a better correlation coefficient of almost $85 \%$, but with the average error up to $16 \%$. The last two methods are obviously unsuccessful in reproducing the average breakthrough time.

Fig. 10 gives a comparison of the all five breakthrough curves from a typical realization. The NP method fails to reproduce the result of the reference field. The proposed method has a better result than the GM method, especially at the late arrival time for this typical realization.

Fig. 11 shows the comparison of the breakthrough curve matching with reference fields between the proposed method and the GM method. As for the average breakthrough error, the proposed approach has a quite noticeable gain over the GM method. All four sample points of the former have closer values to the reference ones than those of

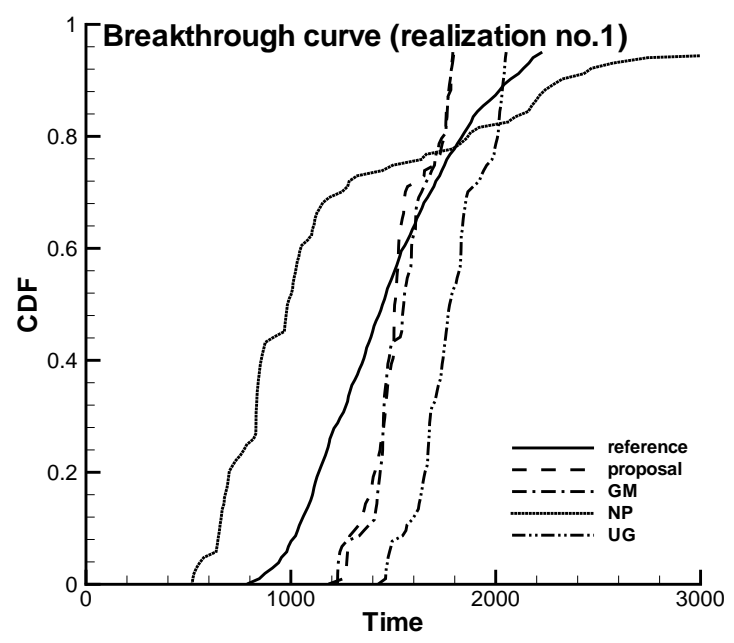

Fig. 6 Typical breakthrough curves of the fine scale and the coarse scales of multi-Gaussian transmissivity field with isotropic structures. 

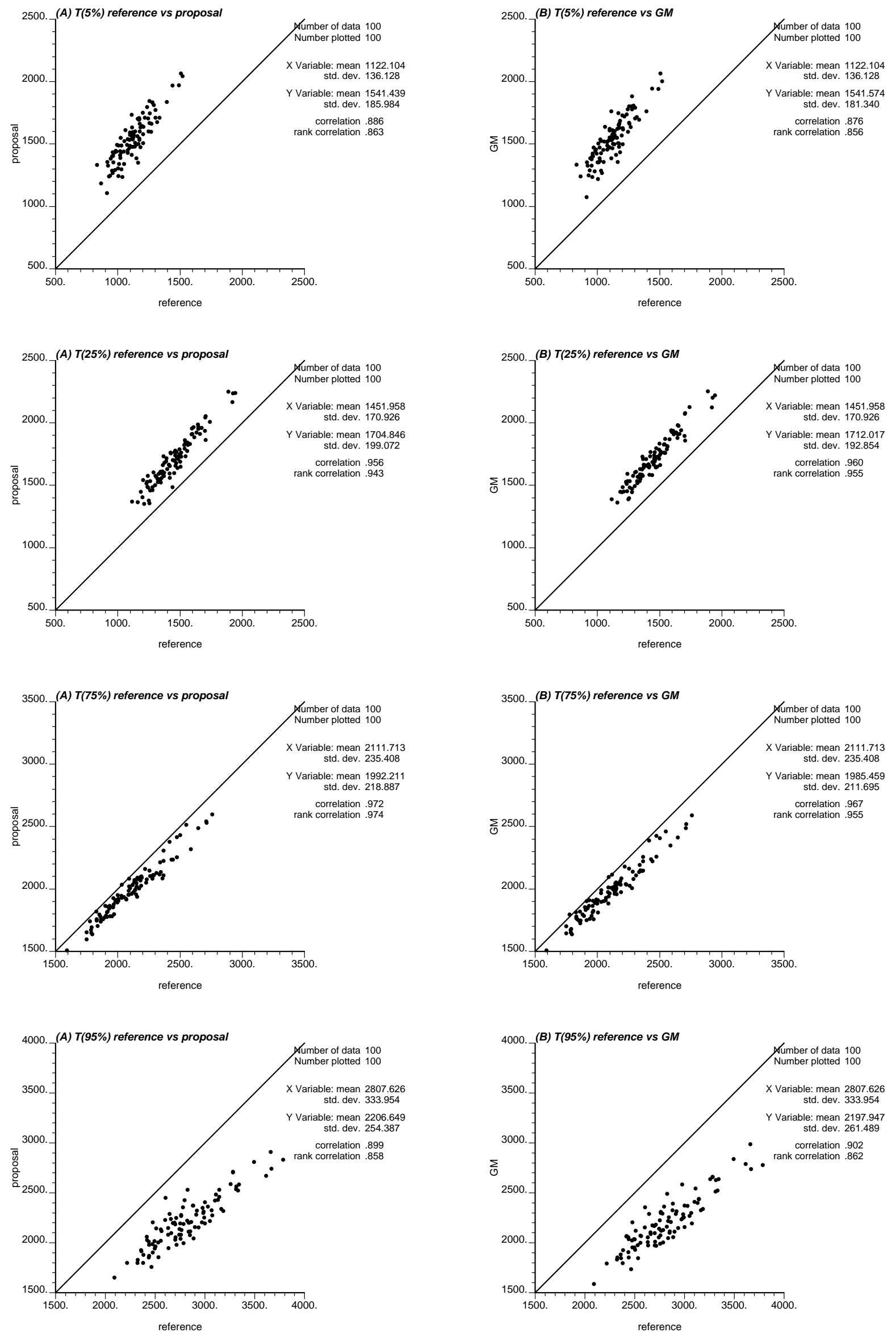

Fig. 7 Comparison of breakthrough curve reproduction of multi-Gaussian transmissivity fields with isotropic structures: (a) the proposed method (left column), (b) the geometrical mean (right column). 


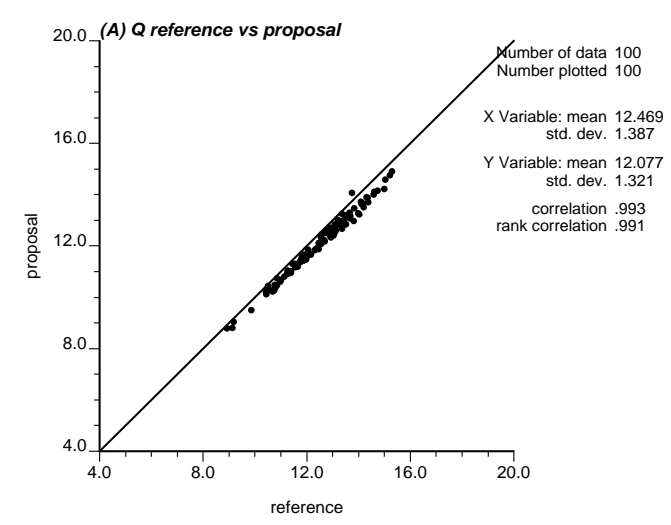

(a) Q reference vs proposal

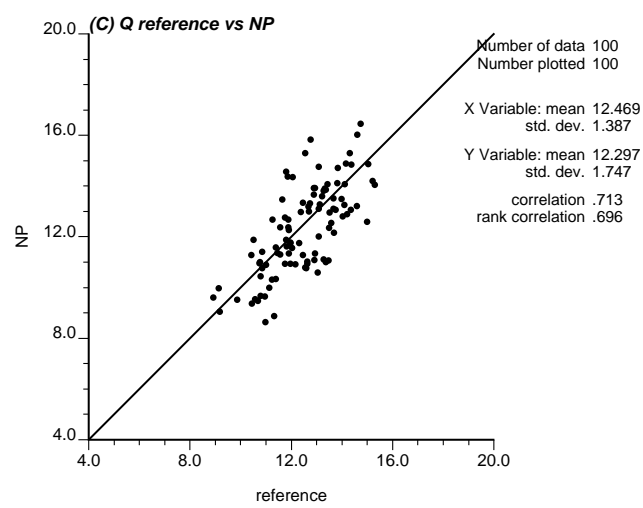

(c) Q reference vs NP

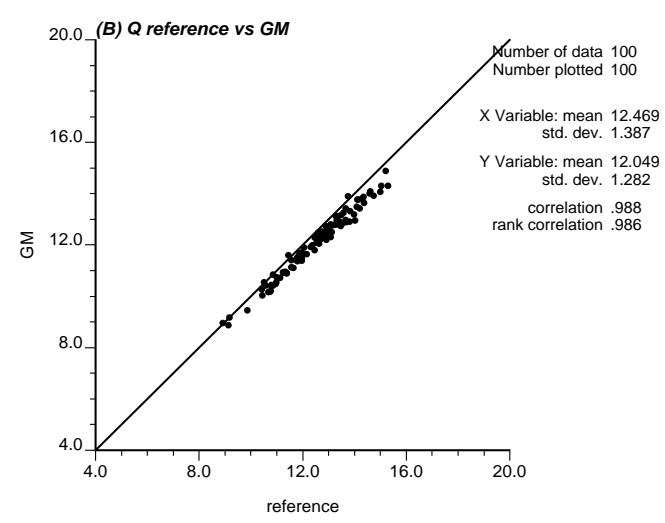

(b) Q reference vs GM

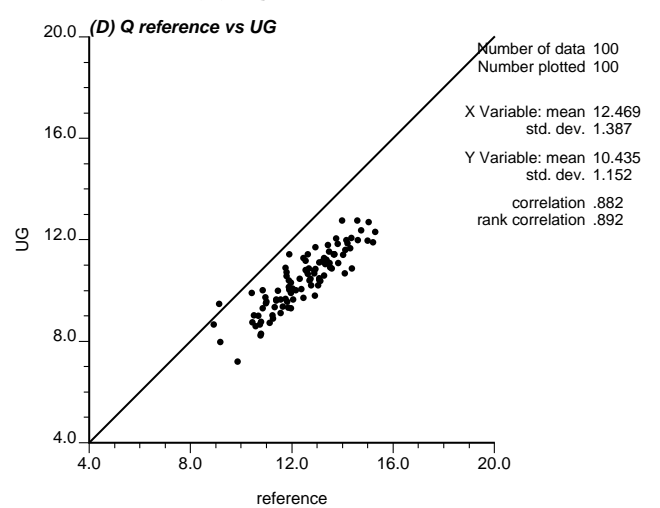

(d) Q reference vs UG

Fig. 8 Wellbore fluxes cross relationship between the fine scale and the coarse scale of multi-Gaussian transmissivity fields with anisotropic structures.

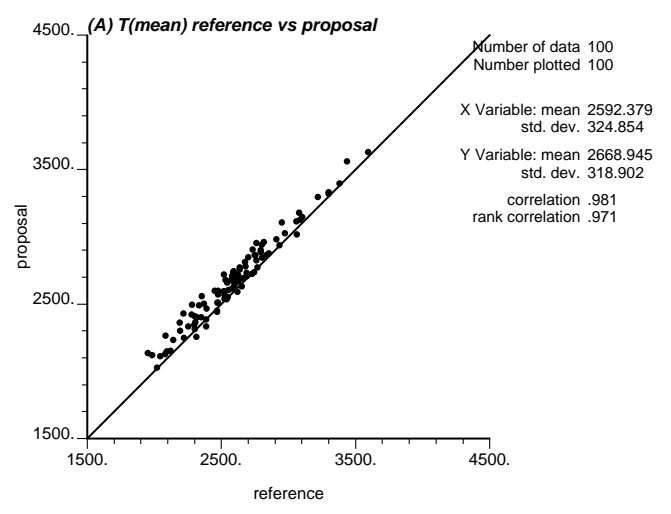

(a) T(mean) reference vs proposal

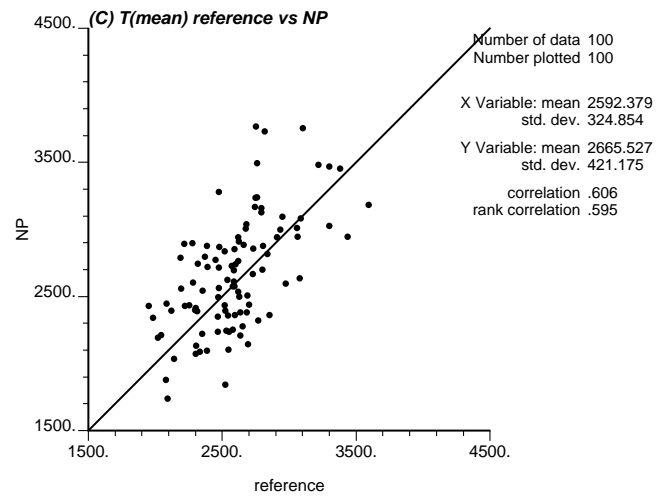

(c) T(mean) reference vs NP

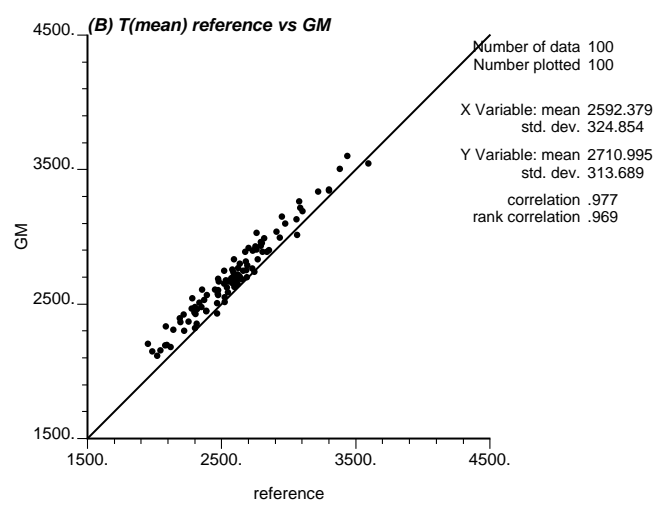

(b) T(mean) reference vs GM

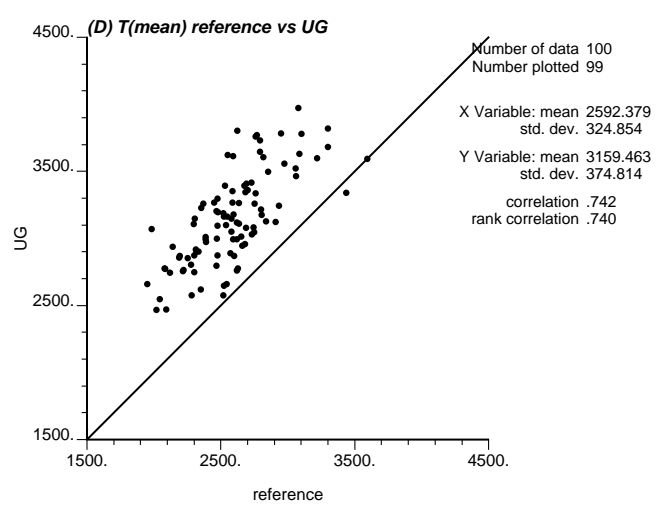

(d) T(mean) reference vs UG

Fig. 9 Average breakthrough time cross relationship between the fine scale and the coarse scale of multi-Gaussian transmissivity fields with anisotropic structures. 


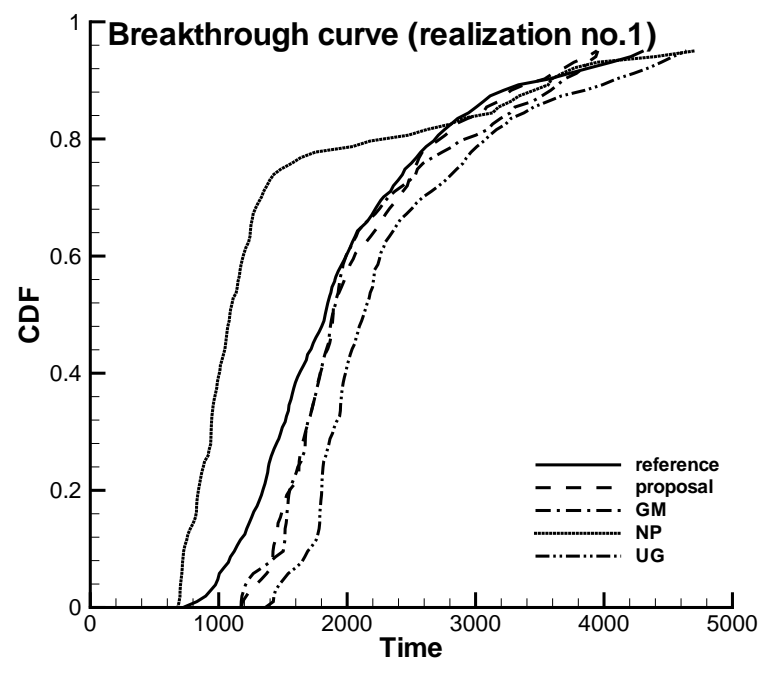

Fig. 10 Typical breakthrough curves of the fine scale and the coarse scales of multi-Gaussian transmissivity field with anisotropic structures.

the latter. The error reduction is $16.6 \%$ for the first point $t_{5 \%}, 39.9 \%$ for the second $t_{25 \%}, 25.6 \%$ for the third $t_{75 \%}$, and $51.4 \%$ for the fourth $t_{95 \%}$. This enhancement is not observed so clearly as in the case of isotropic structure. Moreover the stability of the proposal has no decrease. The correlation coefficients are over $90 \%$ for all sample points. This is true especially for reproducing the early arrival particles.

\subsection{Non-Gaussian fields with anisotropic structures}

One hundred $\ln K(\mathbf{x})$ fields are generated by ISIM3D, each of them with a correlation length equal to ten in the $x$ direction and three in the $y$ direction, i.e., $\lambda_{x}=10$ and $\lambda_{y}=3$. The mean and variance of $\ln K(\mathbf{x})$ is set to zero and two, i.e., $\mathrm{E}[\ln K(\mathbf{x})]=0$ and $\sigma_{\ln K(\mathbf{x})}{ }^{2}=2$. The $\ln K(\mathbf{x})$ fields are constant vectorial, that is, the $\ln K(\mathbf{x})$ values in the $y$ direction are one half of those in the $x$ direction, i.e., $\ln K_{x x}=\ln K_{y y} / 2$. The fluxes over the wellbore are computed for the fine-scale field and four different coarse fields in the same manner as in the first scenario.

Fig. 12 shows the cross relationship of wellbore fluxes between the reference and four different upscaling approaches. Fig. 12(a) exhibits one hundred wellbore fluxes via the proposed upscaling approach comparing to those of reference fine scales. The average flux of one

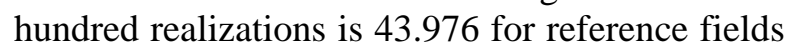
and 35.292 for the proposed method with a relative error of $19.7 \%$. The correlation and rank correlation coefficient between the two types of fluxes are more than 99\%, which demonstrates the robustness of this approach. Fig. 12(b) indicates the performance of the traditional geometric mean method. The correlation and rank correlation coefficient have a little decrease but still close to 99\%. Fig. 12(c) plots the result of the NP method. The average flux of one hundred realizations is 43.976 for reference fields and 42.483 for the proposed method with a relative error of $3 \%$. The correlation coefficient is still up to $90 \%$. Fig. 12(d) is the results of the UG method which distinctly underestimates the actual values.

Fig. 13 plots the average breakthrough times of different upscaled fields versus those of reference fields with anisotropic structures. Fig. 13(a) shows the average breakthrough times of one hundred realizations from the proposed upscaling approach. The correlation coefficient with those of the reference fields is up to $97 \%$ and the relative average error is only $1.4 \%$. The GM method also provides acceptable results as shown in Fig. 13(b). The relative average error is 9.3\% and the correlation coefficient is a little bit worse but still more than $97 \%$. The NP method produces unpleasant results as in Fig. 13(c). The UG method has the results almost the same as those of the NP method. The last two methods are obviously unsuccessful in reproducing the average breakthrough time.

Fig. 14 gives a comparison of the all five breakthrough curves from a typical realization. Due to the peculiarity of non-Gaussian field, there is no upscaling method capable of reproducing the reference field completely. The results of the four coarse girds, as shown in this typical realization, obviously deviate from those of the reference fine grid. But generally, the proposed method produces a better result than the others.

Fig. 15 shows the comparison of the breakthrough curve matching with reference fields between the proposed method and the GM method. As for the average breakthrough error, the proposed approach has a quite noticeable gain over the GM method. All four sample points of the former have closer values to the reference ones than those of the latter. The error reduction is $47 \%$ for the first point $\left(\mathrm{t}_{5 \%}\right), 63.3 \%$ for the second $\left(\mathrm{t}_{25 \%}\right), 37.3 \%$ for the third $\left(\mathrm{t}_{75 \%}\right)$, and $83.4 \%$ for the fourth $\left(\mathrm{t}_{95 \%}\right)$. This enhancement is more obvious than that of multi-Gaussian fields.

\section{DISCUSSION AND CONCLUSIONS}

This paper compares two types of geostatistical techniques for generating hydraulic conductivity fields at the near-well region by assuming spatial structures. One creates property fields directly on 

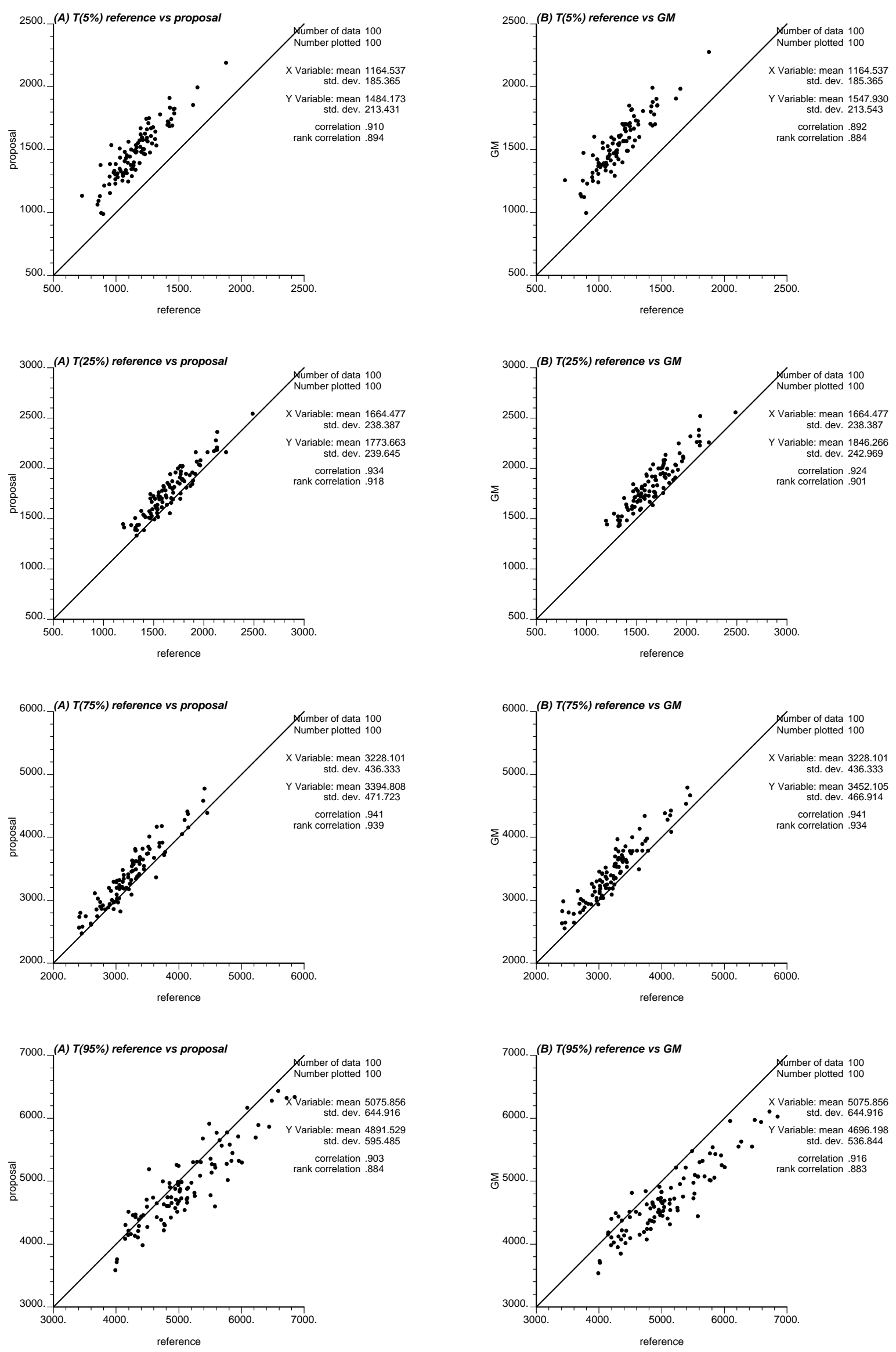

Fig. 11 Comparison of breakthrough curve reproduction of multi-Gaussian transmissivity fields with anisotropic structures: (a) the proposed method (left column), (b) the geometrical mean (right column). 


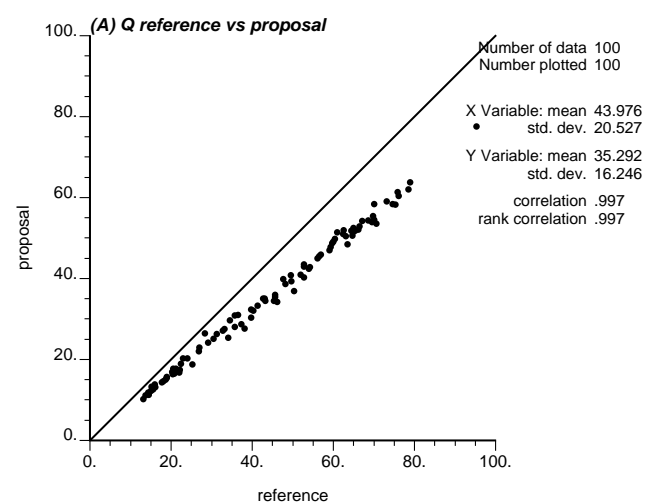

(a) Q reference vs proposal

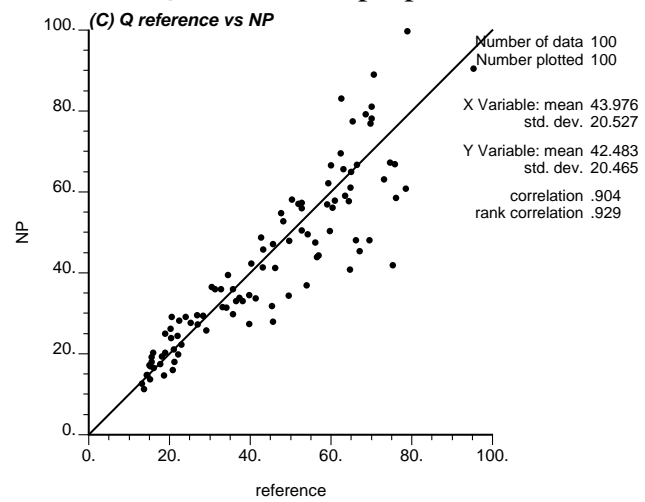

(c) Q reference vs NP

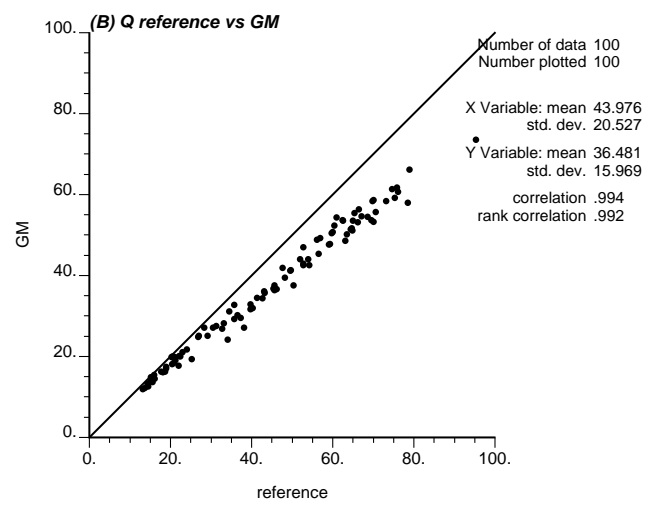

(b) Q reference vs GM

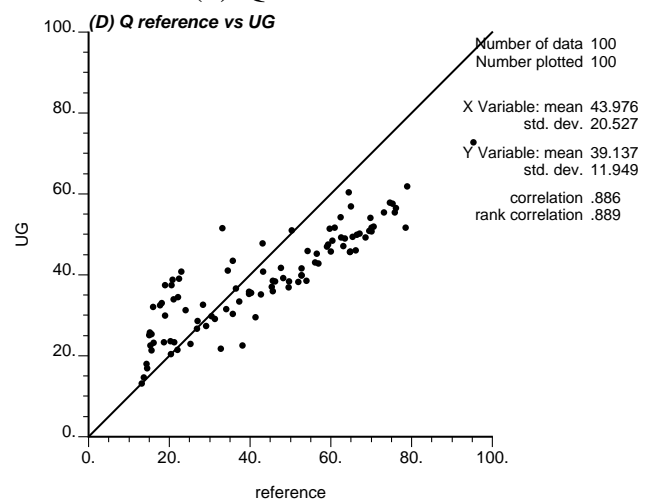

(d) Q reference vs UG

Fig. 12 Wellbore fluxes cross relationship between the fine scale and the coarse scale of non-Gaussian transmissivity fields with anisotropic structures.

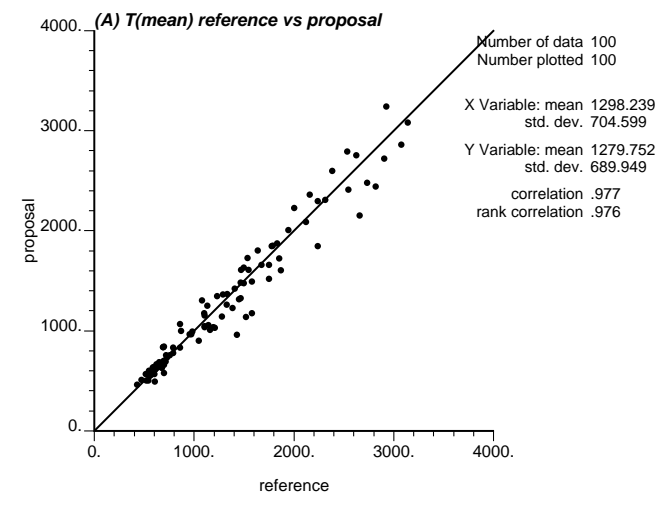

(a) T(mean) reference vs proposal

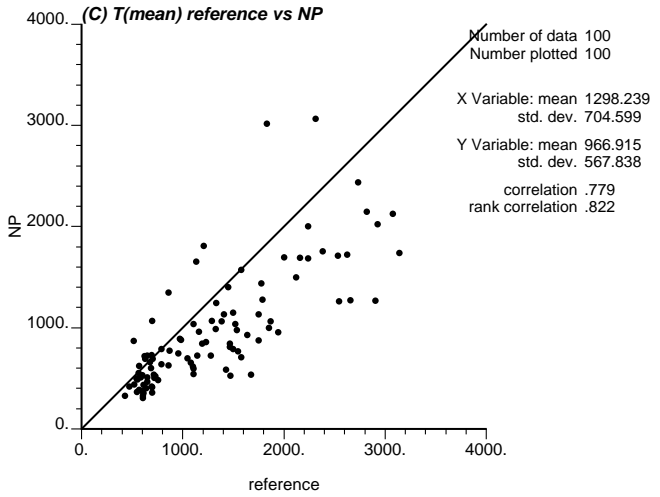

(c) T(mean) reference vs NP

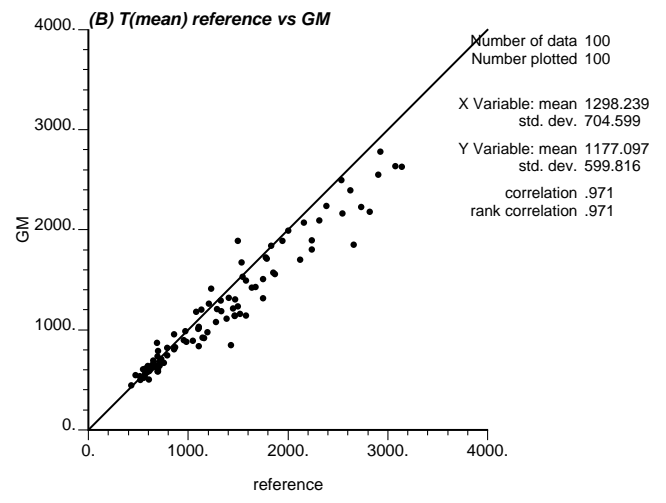

(b) T(mean) reference vs GM

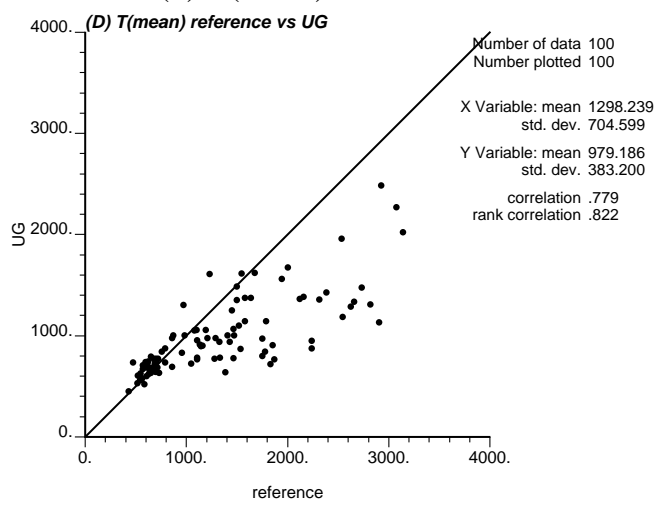

(d) T(mean) reference vs UG

Fig. 13 Average breakthrough time cross relationship between the fine scale and the coarse scale of non-Gaussian transmissivity fields with anisotropic structures. 


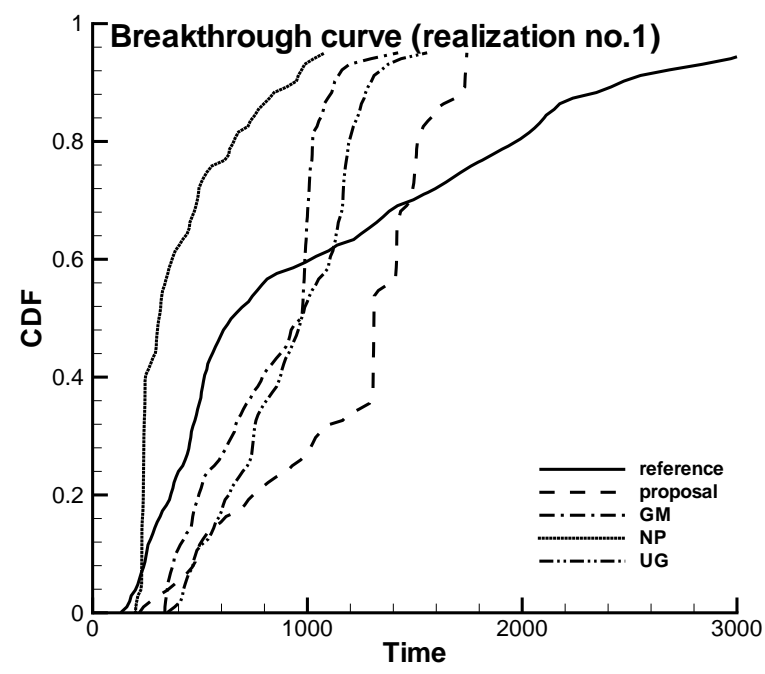

Fig. 14 Typical breakthrough curves of the fine scale and the coarse scales of non-Gaussian transmissivity field with anisotropic structures.

the coarse scale and the other reconstructs such fields via upscaling from the corresponding fine scale. The results from flow and transport simulations demonstrate that the directly generated field can not effectively capture the spatial variations of hydraulic conductivity while the upscaled field can do. Therefore, the requirement of upscaling in the near-well region is apparent.

Previous studies indicate that the flow to a well is strongly influenced by local conditions around the wellbore and is less influenced by fluctuations far away from the well, e.g., Sanchez-Vila et al. (1997) and Axness and Carrera (1999). At the radial flow zone near the wellbore, therefore, one should adopt a different upscaling scheme than that of the linear flow zone far away from the well in order to correctly answer this response. This study shows that the non-uniform radial coarse grid can effectively capture this character.

We further extend the upscaling approach of Gomez-Hernandez and Journel (1994), which was proved efficient under the uniform flow condition, to the convergent or divergent flow case by designing proper radial grids that sufficiently account for the flow patterns in the near-well region. The proposed scheme gains some improvement over the simple geometric mean. Several stochastic models with a single well system are chosen to illustrate the efficiency and robustness of this method.

Numerical simulations from mass transport demonstrate that the simple geometric mean can reasonably reproduce the results of the reference fine scale for multi-Gaussian models, either statistically isotropic or weakly anisotropic. But it fails to do so for non-Gaussian models. The multi-Gaussian model implies the minimal spatial correlation of extreme values, which is critical for mass transport and may be in contradiction with some geological reality, e.g., channeling. Connectivity patterns of extreme conductivity values can not be represented by a multi-Gaussian model. Gomez-Hernandez and Wen (1998) proved that the groundwater travel time predicted by the multi-Gaussian model could be ten times slower than that by non-Gaussian models. The reason is that, for a non-Gaussian model, the simple geometric mean weakens the heterogeneity of conductivity field while the proposed upscaling method effectively preserves such extreme values, with high connectivity either at the extremely high values or at the extremely low values, by solving local flow problems. On the other hand, for a multi-Gaussian model where the spatial variability is not so high as in the nonGaussian model, the simple geometric mean can produce quite similar results as the proposed approach. We notice that the proposed method has not sufficiently reproduced the result of the reference field. A promising improvement is to use full tensorial transmissivity fields, i.e., with the introduction of $T_{V, x y}$ and $T_{V, y x}$.

Finally, several conclusions from this study are worth repeating as follows: (1) Upscaling transmissivity in the near-well region can efficiently preserve the main features of flow and transport in the heterogeneous media. (2) The proposed method improves the ability of predicting well discharge or recharge and solute transport in terms of the coarse grid. Several synthetical examples prove that the proposed upscaling approach is efficient and robust both for flow simulation and for transport simulation. (3) The geometric mean of log conductivity is an alternative approach in upscaling transmissivity in the near-well region. This is true especially when the log-conductivity is a multi-Gaussian field. (4) Uniform gird fails to capture the flow and transport features in the near-well region.

\section{REFERENCES}

1. Axness CL, Carrera J (1999). The 2D steady hydraulic head field surrounding a pumping well in a finite heterogeneous confined aquifer. Mathematical Geology 31(7):873906. 

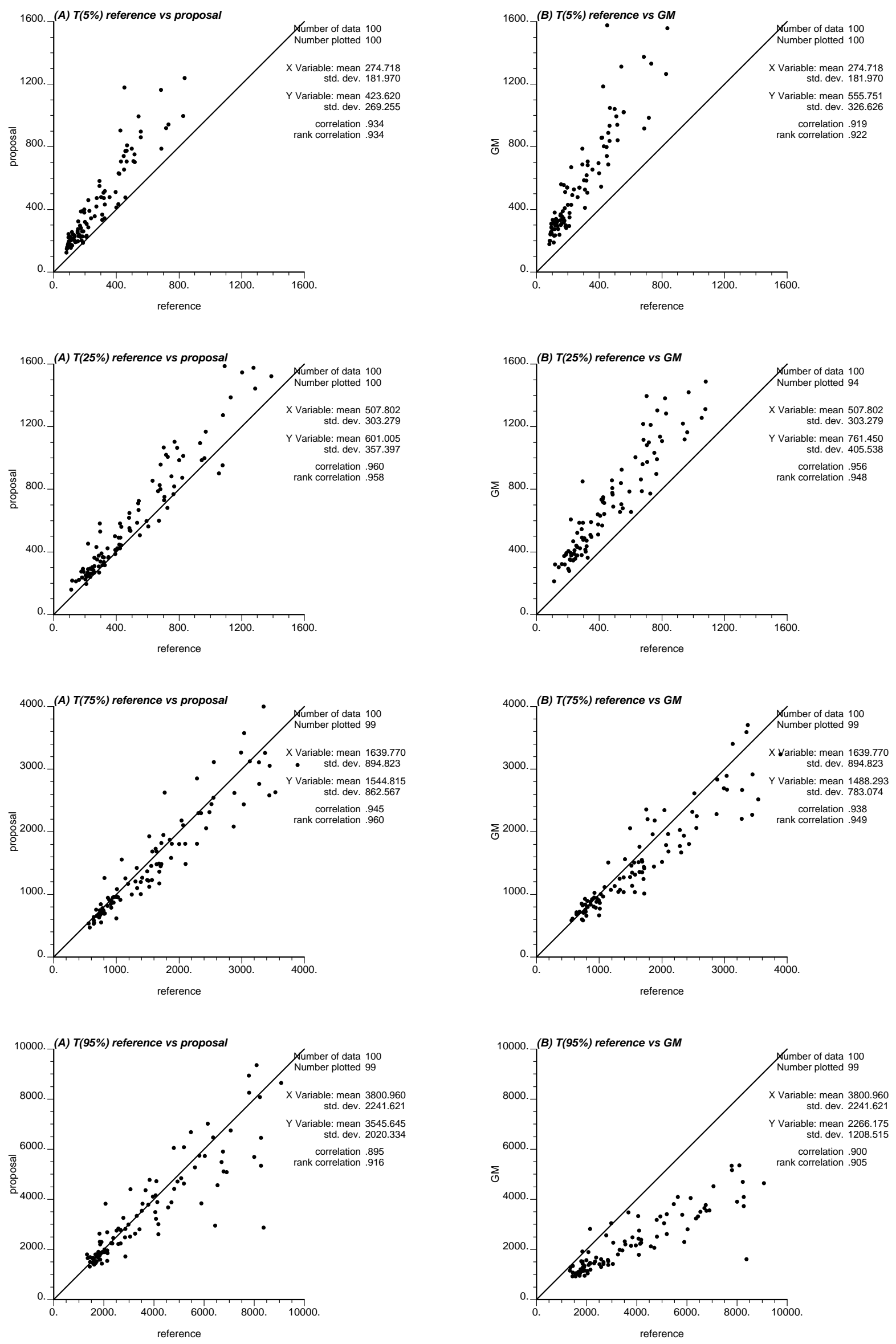

Fig. 15 Comparison of breakthrough curve reproduction of non-Gaussian transmissivity fields with anisotropic structures: (a) the proposed method (left column), (b) the geometrical mean (right column). 
2. Axness CL, Carrera J, Bayer M (2004). Finite-element formulation for solving the hydrodynamic flow equation under radial flow conditions. Computers \& Geosciences 30(6):663-670.

3. Chen Y, Durlofsky LJ, Gerritsen M, Wen X$H$ (2003). A coupled local-global upscaling approach for simulating flow in highly heterogeneous formations. Advances in Water Resources 26(10):1041-1060.

4. Desbarats AJ (1992). Spatial averaging of transmissivity in heterogeneous fields with flow toward a well. Water Resources Research 28(3):757-767.

5. Ding Y (1995). Scaling-up in the vicinity of wells in heterogeneous field, paper SPE 29137 presented at the 1995 SPE Symposium on Reservoir Simulation, San Antonio. Feb. 12-15.

6. Durlofsky LJ (1991). Numerical calculation of equivalent grid block permeability tensors for heterogeneous porous media. Water Resources Research 27(5):699-708.

7. Durlofsky LJ, Jones RC, Milliken WJ (1997). A nonuniform coarsening approach for the scale-up of displacement processes in heterogeneous porous media. Advances in Water Resources 20(5-6):335-347.

8. Durlofsky LJ, Milliken WJ, Bernath A (2000). Scaleup in the near-well region. SPEJ 5:110117.

9. Fiori A, Indelman P, Dagan G (1998) Correlation structure of flow variables for steady flow toward a well with application to highly anisotropic heterogeneous formations. Water Resources Research 34(4):699-708.

10. Fu J (2008). A Markov Chain Monte Carlo Method for Inverse Stochastic Modeling and Uncertainty Assessment, Unpublished Ph.D. Thesis, Universidad Politecnica de Valencia, Valencia, Spain, pp.140.

11. Fu J, Gomez-Hernandez JJ (2008). Preserving spatial structure for inverse stochastic simulation using blocking Markov chain Monte Carlo method. Inverse Problem in Sciences and Engineering 16(7):865-884.

12. Fu J, Gomez-Hernandez JJ (2009a). A blocking Markov chain Monte Carlo method for inverse stochastic hydrogeological modeling. Mathematical Geosciences 41(2):105-128.

13. Fu J, Gomez-Hernandez JJ (2009b). Uncertainty assessment and data worth in groundwater flow and mass transport modeling using a blocking Markov chain
Monte Carlo method. Journal of Hydrology 364:328-341.

14. Fu J, Gomez-Hernandez JJ, Axness CL (2006). Upscaling transmissivity in the nearwell region, Calibration and Reliability in Groundwater Modeling: From Uncertainty to Decision Making, IAHS Publ. 304. pp.209214.

15. Fu J, Tchelepi HA, Caers J (2010). A multiscale adjoint method to compute sensitivity coefficients for flow in heterogeneous porous media. Advances in Water Resources 33(6):698-709.

16. Gomez-Hernandez JJ, Journel AG (1993). Joint sequential simulation of multi-Gaussian fields, in Geostatistics Troia '92, ed. A. Soares, Vol.1. Kluwer, Dordrecht. pp.85-94.

17. Gomez-Hernandez JJ, Journel AG (1994). Stochastic characterization of gridblock permeabilities. SPE Formation Evaluation pp.93-99.

18. Gomez-Hernandez JJ, Srivastava RM (1990) ISIM3D: an ANSI-C three-dimensional multiple indicator conditional simulation program. Computers \& Geosciences 16(4):395-440.

19. Gomez-Hernandez JJ, Wen X-H (1998). To be or not to be multi-Gaussian? A reflection on stochastic hydrology. Advances in Water Resources 21(1):47-61.

20. Holden L, Nielson BF (2000). Global upscaling of permeability in heterogeneous reservoirs: the output least squares (OLS) method. Transport Porous Media 40:115-143.

21. Jenny P, Lee SH, Tchelepi HA, 2003. Multiscale finite-volume method for elliptic problems in subsurface flow simulation, Journal of Computational Physics 187:47-67.

22. Li H, Ranjith PG, Yamaguchi S, Sato M (2007). Development of a 3D FEM simulator on multiphase seepage flows and its applications, Engineering Applications of Computational Fluid Mechanics 1(3):227237.

23. Mascarenhas O, Durlofsky LJ (2000). Coarse scale simulation of horizontal wells in heterogeneous reservoirs. Journal of Petroleum Science and Engineering 25:135147.

24. Muggeridge $A H$, Cuypers $M$, Bacquet $C$, Barker JW (2002). Scale-up of well performance for reservoir flow simulation. Petroleum Geoscience 8(2):133-139.

25. Renard P, de Marsily G (1997). Calculating equivalent permeability: a review. Advances in Water Resources 20(5-6):253-278. 
26. Sanchez-Vila X (1997). Radially convergent flow in heterogeneous porous media. Water Resources Research 33(7):1633-1641.

27. Wagner BJ, Gorelick SM (1989). Reliable aquifer remediation in the presence of spatial variable hydraulic conductivity: from data to design. Water Resources Research 25(10):2211-2225.

28. Wen X-H, Durlofsky LJ, Edwards MG (2003). Use of border regions for improved permeability upscaling. Mathematical Geology 35(5):521-547.

29. Wen X-H, Gomez-Hernandez JJ (1996). Upscaling hydraulic conductivities in heterogeneous media: an overview. Journal of Hydrology 183(1-2):R9-R32.

30. White CD, Horne RN (1987). Computing absolute transmissivity in the presence of fine-scale heterogeneity, SPE 16011.

31. Wolfsteiner C, Durlofsky LJ (2002). Nearwell radial upscaling for the accurate modeling of nonconventional wells, SPE76779. SPE Western Regional/AAPG Pacific Section Joint Meeting held in Anchorage, Alaska, U.S.A., 20-22 May. 